Cite this: J. Mater. Chem. C, 2014, 2, 3756

Received 21st January 2014

Accepted 21st February 2014

DOI: $10.1039 / c 4 t c 00145 a$

www.rsc.org/MaterialsC

\section{Crafting NPB with tetraphenylethene: a win-win strategy to create stable and efficient solid-state emitters with aggregation-induced emission feature, high hole-transporting property and efficient electroluminescence $\dagger$}

\begin{abstract}
Wei Qin, ${ }^{\text {ab }}$ Jianzhao Liu, ${ }^{\text {ab }}$ Shuming Chen, ${ }^{c}$ Jacky W. Y. Lam, ${ }^{\text {ab }}$ Mathieu Arseneault, ${ }^{\text {ab }}$ Zhiyong Yang, ${ }^{\text {ab }}$ Qiuli Zhao, ${ }^{d}$ Hoi Sing Kwok ${ }^{c}$ and Ben Zhong Tang ${ }^{\star a b e}$

$N, N^{\prime}$-Di-(1-naphthyl)- $N, N^{\prime}$-diphenyl-(1,1'-biphenyl)-4,4'-diamine (NPB) possesses high thermal and morphological stability and is one of the well-known hole-transporting materials for the fabrication of organic light-emitting diodes (OLEDs). Modification of NPB by the covalent integration of tetraphenylethene (TPE) into its structure dramatically changes its emission behavior: the resulting adduct (TPE-NPB) is highly emissive in the aggregated state, showing a novel phenomenon of aggregation-induced emission (AIE). The adduct is thermally and morphologically stable. Non-doped multilayer electroluminescence (EL) devices using TPE-NPB as an emitting layer were fabricated, which emitted green light with a maximum luminance and current efficiency of $11981 \mathrm{~cd} \mathrm{~m}^{-2}$ and $11.9 \mathrm{~cd} \mathrm{~A} \mathrm{~A}^{-1}$, respectively. Even better device performances are observed in the bilayer device without NPB. Our strategy takes the full advantage of the AIE property in the solid state and retains the inherent properties of conventional luminophores. It opens a new avenue in the development of stable and efficient solidstate fluorescent materials for OLED application.
\end{abstract}

\section{Introduction}

Development of efficient luminescent materials in the solid state is important for various photonic applications such as organic light-emitting diodes (OLEDs) ${ }^{1}$ and transistors, ${ }^{2}$ organic lasers, ${ }^{3}$ and fluorescent sensors or probes. ${ }^{4}$ However, many conventional $\pi$-conjugated fluorophores are highly emissive in solutions but become faintly emissive in the condensed phase due to the severe intermolecular interaction, ${ }^{5}$ which promotes the formation of detrimental species such as excimers and exciplexes. An example of such fluorophores is perylene, in

${ }^{a}$ HKUST-Shenzhen Research Institute, No. 9 Yuexing 1st RD, South Area, Hi-tech Park, Nanshan, Shenzhen, 518057, China.E-mail: tangbenz@ust.hk

${ }^{b}$ Department of Chemistry, Institute for Advanced Study, Division of Life Science, State Key Laboratory of Molecular Neuroscience, Institute of Molecular Functional Materials and Division of Biomedical Engineering, The Hong Kong University of Science \& Technology (HKUST), Clear Water Bay, Kowloon, Hong Kong, China

${ }^{c}$ Center for Display Research, HKUST, Clear Water Bay, Kowloon, Hong Kong, China ${ }^{d}$ Department of Polymer Science and Engineering, Zhejiang University, Hangzhou 310027, China

${ }^{e}$ Guangdong Innovative Research Team, SCUT-HKUST Joint Research Laboratory, State Key Laboratory of Luminescent Materials and Device, South China University of Technology (SCUT), Guangzhou, 51640, China

$\dagger$ Electronic supplementary information (ESI) available. See DOI: 10.1039/c4tc00145a which its efficient blue emission (fluorescence quantum yield in cyclohexane $=94 \%$ ) is almost quenched completely in the aggregated state. ${ }^{6}$ Such aggregation-caused quenching effect (ACQ) has limited and complicated their high-technological applications. Because of this, in optical and electronic devices, they are normally utilized as dopants at low concentration in host materials. ${ }^{7}$ However, to achieve an optimum device performance, careful control on the dopant concentration is required. ${ }^{8}$ Performance degradation due to phase separation upon heating is also a problem. ${ }^{9}$ Thus, an emitting layer made of a single component with no ACQ effect is in urgent demand.

On the other hand, to further enhance the device performance, it is desirable to have emitters with both efficient solidstate emission and good charge-transporting properties. These features lead to simpler fabrication procedures, overall improved device stability and a lower production cost compared to the multilayer OLEDs. ${ }^{10}$ Arylamines, such as $N, N^{\prime}$-bis(3-methylphenyl)- $N, N$-diphenylbenzidine (TPD), are well-known hole-transporting materials. TPD has been frequently utilized in the early OLED study, for its outstanding hole-transporting capability. ${ }^{11}$ However, TPD suffers from a low glass transition temperature $\left(T_{\mathrm{g}}=60^{\circ} \mathrm{C}\right)$, and is found to crystallize at ambient temperature within few hours after vacuum deposition. ${ }^{a}{ }^{a}$ The low morphological stability of TPD greatly decreases the stability and lifetime of the devices. Structural modification by 


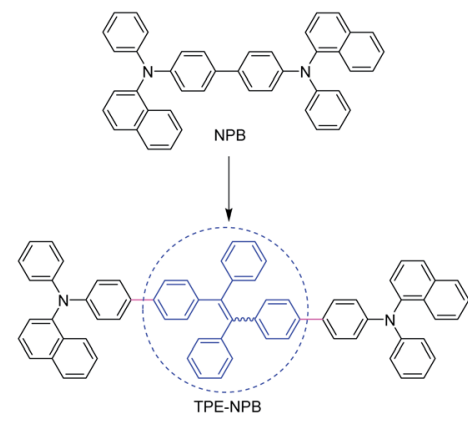

Chart 1 Chemical structures of NPB and TPE-NPB.

replacing two out of the four phenyl rings of TPD attached to the nitrogen atoms by naphthalene rings creates $N, N^{\prime}$-di-(1-naphthyl)- $N, N^{\prime}$-diphenyl-(1,1'-biphenyl)-4, $4^{\prime}$-diamine (NPB) ${ }^{12}$ which retains the high hole mobility of TPD, but exhibits a much enhanced glass transition temperature $\left(T_{\mathrm{g}}=98^{\circ} \mathrm{C}\right)^{7 a}$ and hence a stable thin-film morphology. ${ }^{13}$ Although NPB possesses the abovementioned inherent advantages, it is not a good emitter in the solid state due to $\pi-\pi$ stacking between its molecules. ${ }^{14}$ Thus, it would be nice to create new fluorescent molecules that possess the advantage of NPB but overcome its shortcoming as a non-doped emitter.

Since 2001, we have developed a novel class of organic luminogens with aggregation-induced emission (AIE) characteristics. ${ }^{15}$ These luminogens are non-emissive in dilute solutions but start to luminesce intensely when aggregated. Tetraphenylethene (TPE) is an archetypal AIE luminogen with a simple structure but exhibits a splendid AIE effect. ${ }^{16}$ Incorporation of TPE as a spacer into NPB is anticipated to generate a twisted and nonplanar adduct with properties of both components, i.e. AIE and hole-transporting as well as high thermal and morphological stability (Chart 1).

In this paper, we explored such possibility. As anticipated, TPE-NPB is AIE-active and is thermally and morphologically stable. It possesses a non-planar conformation, which effectively hampers $\pi-\pi$ stacking and endows it with a high fluorescence quantum yield $\left(\Phi_{\mathrm{F}}\right)$ of $98 \%$. Efficient OLEDs using TPE-NPB as the emitter and hole-transporting layer (HTL) are constructed, which exhibit outstanding performances. The performance of the OLED device without NPB as the HTL was even better than that of the device with NPB as the HTL. These results demonstrate a good example for the creation of stable and efficient luminescent materials for OLED application.

\section{Results and discussion}

TPE-NPB was facilely synthesized according to the synthetic routes shown in Scheme 1. The key intermediate 2 was prepared by McMurry coupling of 4-benzoyl-4'-bromobiphenyl catalyzed by $\mathrm{TiCl}_{4} / \mathrm{Zn}$ in reflux tetrahydrofuran (THF). Amination of 2 with $N$-phenyl-1-naphthylamine, a commercially available chemical, was mediated by $\operatorname{Pd}(\mathrm{OAc})_{2}$ in a basic medium, furnishing the desired product TPE-NPB in a satisfactory yield. We have previously prepared a similar compound (2TPATPE) from TPE

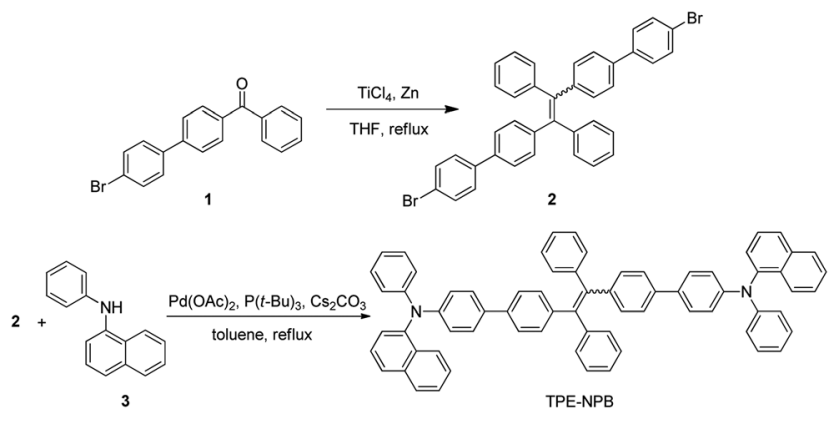

Scheme 1 Synthetic routes to TPE-NPB.

and triphenylamine but its synthesis involves a more complicated procedure. ${ }^{10 b}$ All the intermediates and product were characterized by standard spectroscopic techniques, from which satisfactory analysis data corresponding to their chemical structures were obtained. Details are provided in the Experimental section and examples of spectra are shown in Fig. S1 and S2 in the ESI. $\uparrow$ TPE-NPB shows a good solubility in common organic solvents, such as toluene, dichloromethane, chloroform and THF, but is insoluble in water. The absorption spectra of NPB and TPE-NPB in THF are peaked at $339 \mathrm{~nm}$ and $340 \mathrm{~nm}$, respectively, indicative of a similar conjugation between the two molecules (Table 1).

We first investigated the photoluminescence (PL) of NPB in THF and its mixture with water. The dilute THF solution of NPB emits a blue fluorescence at $\sim 450 \mathrm{~nm}$. Addition of water into the THF solution has slightly weakened the light emission and shifted the emission maximum to $\sim 460 \mathrm{~nm}$ (Fig. 1). The emission becomes strong again when the water content exceeds $70 \%$, and overall a slight PL enhancement was observed.

The effect of water addition on the PL property of TPE-NPB in THF is more obvious. As shown in Fig. 2, the PL spectrum of TPE-NPB is basically a flat line parallel to the abscissa in pure THF. The spectral pattern remains unchanged at water content up to $60 \%$. Afterwards, the PL intensity rises swiftly. The higher the water fraction, the stronger is the emission intensity. The emission intensity reaches its maximum value at $90 \%$ water content, which is 96-fold higher than that in pure THF solution. This indicates that the PL of TPE-NPB is enhanced by aggregate formation, or in other words, it is AIE-active. Measurements of its fluorescence quantum yields in the solution and solid states also support the above conclusion. The $\Phi_{\mathrm{F}}$ in pure THF is merely

Table 1 Optical and thermal properties of NPB and TPE-NPB ${ }^{a}$

\begin{tabular}{llllllll}
\hline & & \multicolumn{5}{l}{$\lambda_{\text {em }} / \mathrm{nm}$} \\
\cline { 5 - 6 } Compound & $\lambda_{\mathrm{ab}} / \mathrm{nm}$ & $E_{\mathrm{g}} / \mathrm{eV}$ & Soln & Aggr & Film & $T_{\mathrm{d}} / T_{\mathrm{g}} /{ }^{\circ} \mathrm{C}$ \\
\hline NPB & 339 & 3.0 & 449 & 443 & 443 & $435 / 98^{b}$ \\
TPE-NPB & 340 & 3.02 & 511 & 505 & 506 & $466 / 149$
\end{tabular}

${ }^{a}$ Abbreviation: $\lambda_{\mathrm{ab}}=$ absorption maximum in THF, $E_{\mathrm{g}}=$ energy band gap calculated from the onset of the absorption spectrum, $\lambda_{\mathrm{em}}=$ emission maximum in THF solution (soln), THF-water mixture (1:9 by volume) (aggr) and solid thin film, $T_{\mathrm{d}}=$ temperature for $5 \%$ weight loss, $T_{\mathrm{g}}=$ glass transition temperature. ${ }^{b}$ Data taken from ref. $7 \mathrm{a}$. 

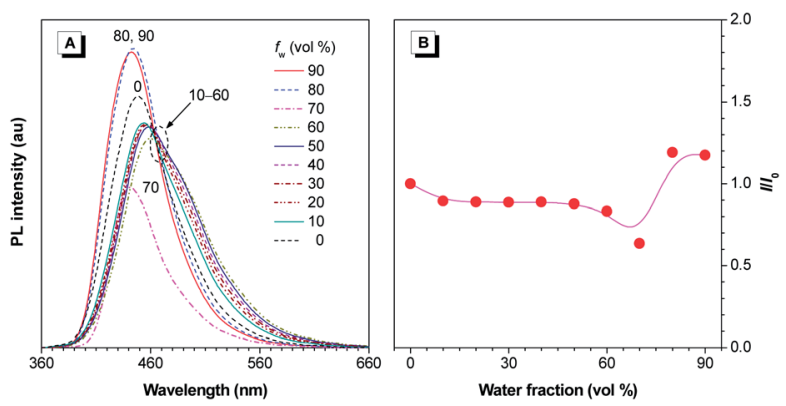

Fig. 1 (A) PL spectra of NPB in THF and water mixtures with different water fractions $\left(f_{\mathrm{w}}\right)$. (B) Plot of the relative $\mathrm{PL}$ intensity $\left(I / I_{0}\right)$ versus the composition of THF-water mixture of NPB. $I_{0}=$ emission intensity in pure THF solution. Concentration: $10 \mu \mathrm{M}$; excitation wavelength: $340 \mathrm{~nm}$.

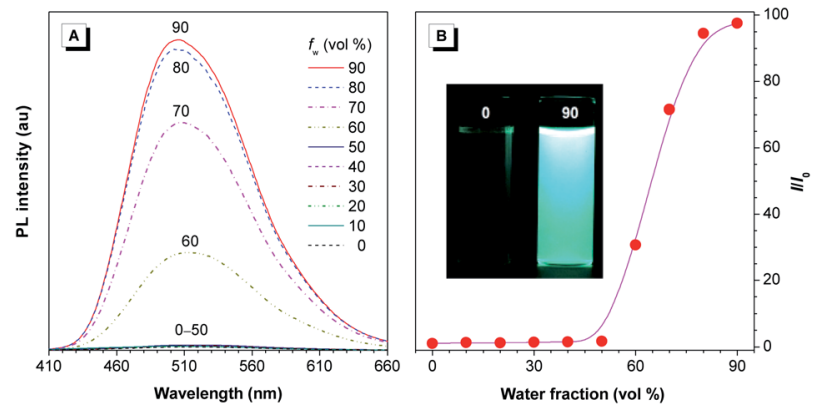

Fig. 2 (A) PL spectra of TPE-NPB in THF and water mixtures with different water fractions $\left(f_{\mathrm{w}}\right)$. (B) Plot of the relative $\mathrm{PL}$ intensity $\left(I / I_{0}\right)$ versus the composition of THF-water mixture of TPE-NPB. $I_{0}=$ emission intensity in pure THF solution. Concentration: $10 \mu \mathrm{M}$; excitation wavelength: $340 \mathrm{~nm}$. Inset in (B) are the fluorescent photographs at $f_{\mathrm{w}}=0$ and $90 \%$ taken under $365 \mathrm{~nm}$ UV irradiation from a handheld UV lamp.

$1.1 \%$, while in the solid state, it reaches $98.0 \%$, which is much higher than those of NPB (26.5\%) and TPE (49.2\%). ${ }^{17}$

The thermal properties of TPE-NPB are investigated by TGA and DSC analyses. As shown in Fig. 3, TPE-NPB shows a high thermal stability, losing $5 \%$ of its weight at $466{ }^{\circ} \mathrm{C}$, which is higher than those of NPB $\left(435{ }^{\circ} \mathrm{C}\right)$ (Fig. S3 $\dagger$ ) and 2TPATPE $\left(361^{\circ} \mathrm{C}\right) . .^{10 b}$

DSC analysis of TPE-NPB in the second heating scan detects a glass transition temperature $\left(T_{\mathrm{g}}\right)$ of $149^{\circ} \mathrm{C}$ (Fig. 3), which is higher than those of NPB $\left(T_{\mathrm{g}}=98^{\circ} \mathrm{C}\right)$ and 2TPATPE $\left(T_{g}=119^{\circ} \mathrm{C}\right) .{ }^{10 b}$ No transition peak associated with crystallization was observed when the fluorophore was heated up to $250{ }^{\circ} \mathrm{C}$. The bulky non-planar TPE unit in TPE-NPB may hinder close packing between molecules in the solid state, thus making the luminogen non-crystalline in nature. Luminogenic materials with high thermal and morphological stability are beneficial to the stability and lifetime of optoelectronic devices. TPENPB possesses such advantages as well as efficient solid-state emission. This makes it promising for the fabrication of efficient EL devices.

The electrochemical property and energy levels of TPE-NPB are investigated by cyclic voltammetry. In our experiment, the working and reference electrodes are glass carbon and

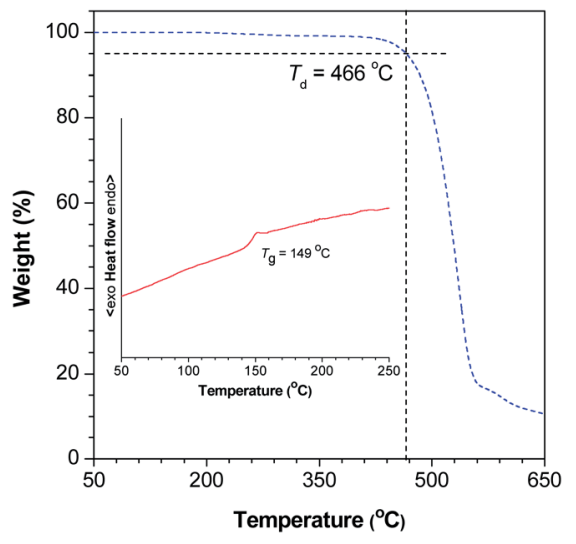

Fig. 3 TGA thermogram of TPE-NPB recorded under nitrogen at a heating rate of $10{ }^{\circ} \mathrm{C} \mathrm{min}^{-1}$. Inset: DSC curve of TPE-NPB recorded during the second heating scan.

$\mathrm{Hg} / \mathrm{HgCl}_{2}$, respectively, with tetra- $n$-butylammonium hexafluorophosphate ( $0.1 \mathrm{M}$ in dichloromethane) as the supporting electrolyte. Using such set-up, ferrocene ( $\left.E_{\text {ferrocene }}\right)$ was found to oxidize at $0.42 \mathrm{~V}$ (Fig. S4 $\dagger$ ), while the oxidation onset potential ( $\left.E_{\text {onset }}\right)$ of TPE-NPB occurs at $0.77 \mathrm{eV}$. Applying the equation: $\mathrm{HOMO}=-\left(E_{\text {onset }}+4.8-E_{\text {ferrocene }}\right) \mathrm{eV}$, the HOMO of TPE-NPB is calculated to be $-5.15 \mathrm{eV}$, which is slightly higher than that of NPB $($ HOMO $=-5.3 \mathrm{eV}) .{ }^{18}$ This suggests that TPE-NPB possesses a good hole-transporting property, presumably due to the fine contribution from the NPB unit. The LUMO of TPE-NPB can be obtained by subtraction of the optical band gap energy $\left(E_{\mathrm{g}}\right)$ estimated from the onset absorption wavelength from the HOMO value and is located at $-2.15 \mathrm{eV}$.

To get further insight into the photophysical property of TPE-NPB, density functional theory calculations are carried out using a suite of the Gaussian 03 program. The nonlocal density functional of B3LYP with 6-31G(d) basis sets was used for the calculation. The optimized structures and orbital distributions of HOMO and LUMO energy levels of TPE-NPB are shown in Fig. 4. TPE-NPB adopts a propeller-like conformation. The torsion angle between the central plane of the TPE core and the adjacent phenyl rings is $\sim 34^{\circ}$. The dihedral angles between any phenyl ring and the vinyl core of the TPE unit are $\sim 50^{\circ}$ (Table $\mathrm{S} 1 \dagger)$. The dihedral angles between any two aryl rings of arylamine substituents in TPE-NPB range from $\sim 63^{\circ}$ to $\sim 83^{\circ}$ (Table $\mathrm{S} 1 \dagger)$. The non-planar conformation of TPE-NPB should prevent close molecular packing in the solid state, leading to efficient fluorescence in the condensed phase. The LUMO of TPE-NPB is dominated by the orbitals from the TPE core. In contrast, the

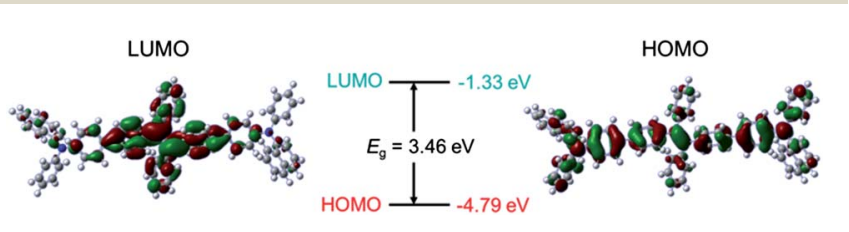

Fig. 4 Optimized geometries and molecular orbital amplitude plots of HOMO and LUMO levels of TPE-NPB calculated using the B3LYP/6$31 \mathrm{G}(\mathrm{d})$ basis set. 

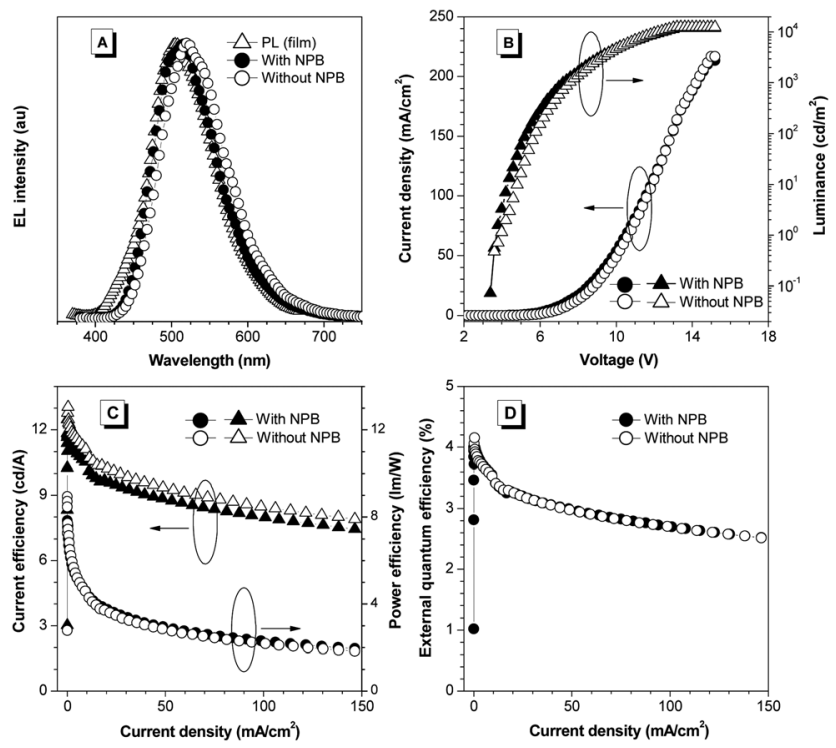

Fig. 5 (A) PL and EL spectra of the solid thin film of TPE-NPB. (B) Current efficiency-voltage-luminance characteristics of a multilayer EL device of TPE-NPB. Changes in (C) power and current and (D) external quantum efficiencies with the applied current density in a multilayer EL device of TPE-NPB. Device configuration: ITO/(NPB)/ TPE-NPB/TPBi/LiF/Al

electron cloud of the HOMO is located mainly on the arylamino functionalities and partly on the TPE core. Such orbital distributions reveal an intrinsic intramolecular charge transfer property of the dye molecule.

The efficient solid emission and good thermal and morphological stability of TPE-NPB prompt us to study its EL
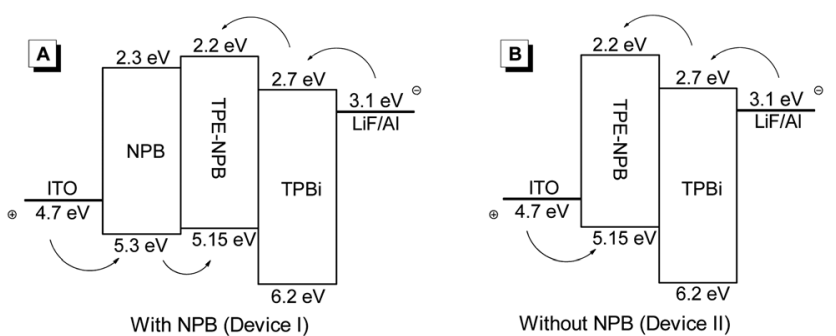

Fig. 6 Energy level diagrams and device configurations of (A) Device I and (B) Device II. Abbreviation: NPB $=N, N^{\prime}$-di-(1-naphthyl)- $N, N^{\prime}-$ diphenyl-(1,1'-biphenyl)-4, $4^{\prime}$-diamine, TPBi $=2,2^{\prime}, 2^{\prime \prime}$ - $(1,3,5$-benzenetriyl)tris(1-phenyl-1- $H$ benzimidazole). property. We first fabricated a single-layer EL device with a configuration of ITO/TPE-NPB $(100 \mathrm{~nm}) / \mathrm{LiF} / \mathrm{Al}(100 \mathrm{~nm})$. The device emits at $516 \mathrm{~nm}$, exhibiting a maximum current density of $1072 \mathrm{~mA} \mathrm{~cm}^{-2}$ and a maximum luminance $\left(L_{\max }\right)$ of $588 \mathrm{~cd}$ $\mathrm{m}^{-2}$ (Fig. S5†). This demonstrates that TPE-NPB possesses a good charge transporting property, even though these results are preliminary. To improve the device performance, we fabricated a multilayer EL device with a configuration of ITO/ $\operatorname{NPB}(40 \mathrm{~nm}) / \mathrm{TPE}-\mathrm{NPB}(20 \mathrm{~nm}) / \mathrm{TPBi}(40 \mathrm{~nm}) / \mathrm{LiF}(1 \mathrm{~nm}) / \mathrm{Al}(100$ $\mathrm{nm})$ (Device I), where NPB functions as a hole-transporting layer (HTL) and 2,2',2'-(1,3,5-benzenetriyl)tris(1-phenyl-1- $H$ benzimidazole) (TPBi) serves as both an electron-transporting and a hole-blocking material. Device I emits at $512 \mathrm{~nm}$, which is slightly red-shifted from the PL peak of TPE-NPB film $(506 \mathrm{~nm})$ but far from the EL peak of NPB $(440 \mathrm{~nm})^{19}$ and TPBi $(400 \mathrm{~nm}){ }^{9 c}$ This confirms that the EL is indeed from the emitting layer. The device turns on at a low bias of $3.7 \mathrm{~V}$, emitting brilliantly with a maximum luminance $\left(L_{\max }\right)$ of $11981 \mathrm{~cd} \mathrm{~m}^{-2}$ and maximum current efficiency $\left(\eta_{\mathrm{C}, \max }\right)$, power efficiency $\left(\eta_{\mathrm{P}, \max }\right)$ and external quantum efficiency $\left(\eta_{\mathrm{ext}, \max }\right)$ of $11.9 \mathrm{~cd} \mathrm{~A}^{-1}, 8.9 \mathrm{~lm} \mathrm{~W} \mathrm{~m}^{-1}$ and $4.0 \%$, respectively (Fig. 5). Such high EL performance is impressive and outperforms the result reported previously. ${ }^{20}$

Since TPE-NPB possesses hole-transporting arylamino moieties, the application of NPB as an additional HTL may not be necessary or can even be harmful because it may break the charge balance in the EL device. From the energy level diagram shown in Fig. 6, it is possible to further enhance the device performance by removing the NPB layer because it will lower the energy gap between the ITO and the HTL or the emitter from 0.6 $\mathrm{eV}$ to $0.45 \mathrm{eV}$. This decreases the charge injection barrier and is beneficial for hole-transportation. To verify this, Device II containing no NPB as an additional HTL was fabricated, which turned on at $3.9 \mathrm{~V}$ and emitted more intensely with a $L_{\max }$ of $12607 \mathrm{~cd} \mathrm{~m}^{-2}$. The $\eta_{\mathrm{C}, \max }, \eta_{\mathrm{P}, \max }$ and $\eta_{\text {ext,max }}$ reach $13.1 \mathrm{~cd} \mathrm{~A}^{-1}$, $7.8 \mathrm{~lm} \mathrm{~W}^{-1}$ and $4.2 \%$, respectively, which are even higher than those of Device I (Table 2). It is noteworthy that the profiles of the EL curves in Device I and II are smooth, which are indicative of their high stability. The above results show that TPE-NPB displays excellent hole transportation and efficient emission in this simplified EL device. Thus, the synthetic procedure adopted in the present work is a win-win strategy, which makes full use of the intrinsic advantages of both TPE and NPB to create AIE luminogens with more efficient solid-state emission, good hole-transporting property and prominent thermal and morphological stability.

Table 2 EL performances of TPE-NPB ${ }^{a}$

\begin{tabular}{|c|c|c|c|c|c|c|c|}
\hline Device & NPB & $\lambda_{\mathrm{EL}} / \mathrm{nm}$ & $V_{\mathrm{on}} / \mathrm{V}$ & $L_{\max } / \mathrm{cd} \mathrm{m}^{-2}$ & $\eta_{\mathrm{C}, \max } / \mathrm{cd} \mathrm{A}^{-1}$ & $\eta_{\mathrm{P}, \max } / \mathrm{lm} \mathrm{\textrm {W } ^ { - 1 }}$ & $\eta_{\text {ext,max }} / \%$ \\
\hline I & $\sqrt{ }$ & 512 & 3.7 & 11981 & 11.9 & 8.9 & 4.0 \\
\hline II & $\times$ & 516 & 3.9 & 12607 & 13.1 & 7.8 & 4.2 \\
\hline
\end{tabular}

${ }^{a}$ Device configuration: ITO/NPB(40 nm)/TPE-NPB(20 nm)/TPBi(40 nm)/LiF(1 nm)/Al(100 nm) (Device I); ITO/TPE-NPB(60 nm)/TPBi(40 nm)/ $\operatorname{LiF}(1 \mathrm{~nm}) / \mathrm{Al}(100 \mathrm{~nm})$ (Device II). Abbreviation: $\lambda_{\mathrm{EL}}=\mathrm{EL}$ maximum, $V_{\mathrm{on}}=$ turn-on voltage at $1 \mathrm{~cd} \mathrm{~m}^{-2}, L_{\max }=$ maximum luminance, $\eta_{\mathrm{P}, \max }=$ maximum power efficiency, $\eta_{\mathrm{C}, \max }=$ maximum current efficiency and $\eta_{\mathrm{ext}, \mathrm{max}}=$ maximum external quantum efficiency. 


\section{Conclusions}

In this paper, TPE-NPB was synthesized and its optical properties were investigated. The dye molecule shows a typical AIE characteristic and improved quantum yields than its parent NPB and TPE molecules. It enjoys high thermal and morphological stability. Non-doped multilayer EL devices are constructed, which show outstanding performances. The bilayer device without NPB as a HTL exhibits even better performances than the one with NPB as a HTL. These results demonstrate that the covalent integration of the AIE unit into conventional chromophores is a good strategy to generate efficient solid-state emitters with novel functional properties. Such method takes the full advantage of AIE property in the solid state and retains other functional properties of the chromophores. Through such strategy, it is expected that new AIE luminogens with new and/or enhanced functional properties will be created.

\section{Experimental}

\section{Materials}

THF was distilled from sodium benzophenone ketyl under dry nitrogen immediately prior to use. NPB was purchased from Luminescence Technology Corp. Other chemicals and reagents were purchased from Aldrich and used as received without further purification.

\section{Instruments}

${ }^{1} \mathrm{H}$ and ${ }^{13} \mathrm{C}$ NMR spectra were recorded on a Bruker AV 300 spectrometer in $\mathrm{CDCl}_{3}$ using tetramethylsilane (TMS; $\delta=0$ ) as an internal reference. High resolution mass spectra (HRMS) were recorded on a GCT premier CAB048 mass spectrometer operating in MALDI-TOF mode. Elemental analysis was performed on a ThermoFinnigan Flash EA1112. UV spectra were recorded on a Milton Roy 5 Spectronic 3000 Array spectrophotometer. Photoluminescence (PL) spectra were recorded on a Perkin-Elmer LS 55 spectrofluorometer. The solution fluorescence quantum yields $\left(\Phi_{\mathrm{F}}\right)$ were determined by using 9,10diphenylanthracene ( $\Phi_{\mathrm{F}}=90 \%$ in cyclohexane) as a standard. The absorbance of the solutions was kept below 0.1 to avoid the internal filter effect. The $\Phi_{\mathrm{F}}$ values of solid thin films were measured by using a calibrated integrating sphere. The thermal properties of TPE-NPB and NPB were evaluated on TA Instruments TGA Q5000 and DSC Q1000 under nitrogen at a heating rate of $10{ }^{\circ} \mathrm{C} \min ^{-1}$.

\section{Preparation of nanoaggregates}

Stock THF solutions of the compounds with a concentration of $10^{-4} \mathrm{M}$ were prepared. Aliquots of the stock solution were transferred to $10 \mathrm{~mL}$ volumetric flasks. After appropriate amounts of THF were added, water was added dropwise under vigorous stirring to furnish $10^{-5} \mathrm{M}$ solutions with different water contents (0-90 vol\%). The PL measurements of the resulting solutions were then performed immediately.

\section{Synthesis of 2}

4-Benzoyl-4'-bromobiphenyl (4.2 g, $12.5 \mathrm{mmol}$ ), Zn dust ( $3.5 \mathrm{~g}$, $54 \mathrm{mmol})$ and THF (125 mL) were added to a $250 \mathrm{~mL}$ two-necked round bottom flask under nitrogen. The solution was cooled to $-78{ }^{\circ} \mathrm{C}$, into which $\mathrm{TiCl}_{4}(4.75 \mathrm{~g}, 25 \mathrm{mmol})$ was added. After refluxing for $36 \mathrm{~h}$, the reaction mixture was cooled to room temperature and concentrated by vacuum. Chloroform $(300 \mathrm{~mL})$ was added to dissolve the product and the mixture was filtered under vacuum suction. The filtrate was concentrated and the crude product was purified on a silica gel column using petroleum ether/chloroform as an eluent. A white solid was obtained in $90 \%$ yield $(3.6 \mathrm{~g}) .{ }^{1} \mathrm{H}$ NMR $\left(300 \mathrm{MHz}, \mathrm{CDCl}_{3}\right), \delta$ (TMS, ppm): 7.52-7.49 (m, 4H), 7.42-7.39 (m, 4H), 7.36-7.29 (m, $4 \mathrm{H}), 7.14-7.04(\mathrm{~m}, 14 \mathrm{H})$. HRMS (MALDI-TOF): $m / z 642.0381\left(\mathrm{M}^{+}\right.$, calcd 642.0381).

\section{Synthesis of TPE-NPB}

Under nitrogen, a mixture of 2 (706 mg, $1.1 \mathrm{mmol}), \mathrm{N}$-phenyl1-naphthylamine $(569.4 \mathrm{mg}, 2.6 \mathrm{mmol}), \mathrm{Cs}_{2} \mathrm{CO}_{3}(2.28 \mathrm{~g}$, $7 \mathrm{mmol}), \mathrm{Pd}(\mathrm{OAc})_{2}(11.2 \mathrm{mg}, 0.05 \mathrm{mmol})$, tri-tert-butylphosphine $(30.3 \mathrm{mg}, 0.15 \mathrm{mmol})$ and toluene $(40 \mathrm{~mL})$ was heated at $40{ }^{\circ} \mathrm{C}$ for $2 \mathrm{~h}$. The reaction mixture was then heated at $110{ }^{\circ} \mathrm{C}$ for $24 \mathrm{~h}$. After the mixture was cooled to room temperature, water $(30 \mathrm{~mL})$ and chloroform $(200 \mathrm{~mL})$ were added. The organic layer was separated, washed with brine, dried over anhydrous $\mathrm{MgSO}_{4}$ and evaporated to dryness under reduced pressure. The crude product was purified by silica gel column chromatography using hexane-chloroform mixture as an eluent, affording a pale yellow solid in $61 \%$ yield $(620 \mathrm{mg}) .{ }^{1} \mathrm{H}$ NMR (300 MHz, $\left.\mathrm{CDCl}_{3}\right), \delta(\mathrm{TMS}, \mathrm{ppm}): 7.92(\mathrm{~d}, J=8.5 \mathrm{~Hz}, 2 \mathrm{H})$, $7.88(\mathrm{~d}, J=8.1 \mathrm{~Hz}, 2 \mathrm{H}), 7.76(\mathrm{~d}, J=8.2 \mathrm{~Hz}, 2 \mathrm{H}), 7.49-7.42(\mathrm{~m}$, $4 \mathrm{H}), 7.39-7.27(\mathrm{~m}, 12 \mathrm{H}), 7.22-7.17(\mathrm{~m}, 4 \mathrm{H}), 7.10-7.00(\mathrm{~m}$, $22 \mathrm{H}), 6.93(\mathrm{t}, J=7.3 \mathrm{~Hz}, 2 \mathrm{H}) .{ }^{13} \mathrm{C}$ NMR $\left(75 \mathrm{MHz}, \mathrm{CDCl}_{3}\right), \delta$ (TMS, ppm): 148.23, 147.67, 143.84, 143.37, 142.18, 140.56, 138.30, 135.26, 133.50, 131.76, 131.43, 131.18, 129.10, 128.38, $127.72,127.61,127.33,127.19,126.49,126.40,126.33,126.13$, $125.58,125.48,124.23,122.10,121.86,121.69,121.67$. HRMS (MALDI-TOF): $\mathrm{m} / z$ 918.3826 $\left(\mathrm{M}^{+}\right.$, calcd 918.3974). Anal. calcd for $\mathrm{C}_{70} \mathrm{H}_{50} \mathrm{~N}_{2}$ : C, 91.47; H, 5.48; N, 3.05\%. Found: C, 91.21; H, $5.66 ; \mathrm{N}, 3.01 \%$.

\section{Acknowledgements}

This work was partially supported by the National Basic Research Program of China (973 Program, 2013CB834701), the Research Grants Council of Hong Kong (HKUST2/CRF/10 and N_HKUST620/11), the Innovation and Technology Commission (ITCPD/17-9), and the University Grants Committee of Hong Kong (AoE/P-03/08 and T23-713/11-1). We thank the support of the Guangdong Innovative Research Team Program (201101C0105067115). We thank Professor Ping Lu in the State Key Laboratory of Supramolecular Structure and Materials of Jilin University for the measurement of fluorescence quantum yields of solid powder samples. 


\section{Notes and references}

1 (a) P. Schouwink, A. H. Schafer, C. Seidel and H. Fuchs, Thin Solid Films, 2000, 372, 163; (b) T. W. Kwon, M. M. Alam and S. A. Jenekhe, Chem. Mater., 2004, 16, 4657.

2 (a) F. Cicoira and C. Santato, Adv. Funct. Mater., 2007, 17, 3421; (b) J. Zaumseil and H. Sirringhaus, Chem. Rev., 2007, 107, 1296.

3 (a) M. D. McGehee and A. J. Heeger, Adv. Mater., 2000, 12, 1655; (b) I. D. W. Samuel and G. A. Turnbull, Chem. Rev., 2007, 107, 1272.

4 (a) S. W. Thomas, G. D. Joly and T. M. Swager, Chem. Rev., 2007, 107, 1339; (b) L. Basabe-Desmonts, D. N. Reinhoudt and M. Crego-Calama, Chem. Soc. Rev., 2007, 36, 993; (c) M. S. Meaney and V. L. McGuffin, Anal. Bioanal. Chem., 2008, 391, 2557; (d) Y. Liu, K. R. Wang, D. S. Guo and B. P. Jiang, Adv. Funct. Mater., 2009, 19, 2230; (e) W. C. Wu, C. Y. Chen, Y. Q. Tian, S. H. Jang, Y. N. Hong, Y. Liu, R. R. Hu, B. Z. Tang, Y. T. Lee, C. T. Chen, W. C. Chen and A. K. Y. Jen, Adv. Funct. Mater., 2010, 20, 1413; $(f)$ W. Qin, D. Ding, J. Z. Liu, W. Z. Yuan, Y. Hu, B. Liu and B. Z. Tang, Adv. Funct. Mater., 2012, 22, 771.

5 (a) S. A. Jenekhe and J. A. Osaheni, Science, 1994, 265, 765; (b) R. H. Friend, R. W. Gymer, A. B. Holmes, J. H. Burroughes, R. N. Marks, C. Taliani, D. D. C. Bradley, D. A. Dos Santos, J. L. Bredas, M. Logdlund and W. R. Salaneck, Nature, 1999, 397, 121; (c) M. Shimizu and T. Hiyama, Chem. - Asian J., 2010, 5, 1516.

6 J. B. Birks, Photophysics of Aromatic Molecules, WileyInterscience, London, 1970.

7 (a) C. H. Chen, J. Shi and C. W. Tang, Macromol. Symp., 1998, 125, 1; (b) W. C. Wu, H. C. Yeh, L. H. Chan and C. T. Chen, Adv. Mater., 2002, 14, 1072.

8 H. C. Yeh, S. J. Yeh and C. T. Chen, Chem. Commun., 2003, 2632.

9 (a) S. C. Chang, G. He, F. C. Chen, T. F. Guo and Y. Yang, Appl. Phys. Lett., 2001, 79, 2088; (b) G. Y. Zhong, Z. Xu, J. He, S. T. Zhang, Y. Q. Zhan, X. J. Wang, Z. H. Xiong, H. Z. Shi, X. M. Ding, W. Huang and X. Y. Hou, Appl. Phys.
Lett., 2002, 81, 1122; (c) J. R. Gong, L. J. Wan, S. B. Lei, C. L. Bai, X. H. Zhang and S. T. Lee, J. Phys. Chem. B, 2005, 109, 1675.

10 (a) S. L. Tao, L. Li, J. S. Yu, Y. D. Jiang, Y. C. Zhou, C. S. Lee, S. T. Lee, X. H. Zhang and O. Y. Kwon, Chem. Mater., 2009, 21, 1284; (b) Y. Liu, S. M. Chen, J. W. Y. Lam, P. Lu, R. T. K. Kwok, F. Mahtab, H. S. Kwok and B. Z. Tang, Chem. Mater., 2011, 23, 2536.

11 (a) M. Stolka, J. F. Yanus and D. M. Pai, J. Phys. Chem., 1984, 88, 4707; (b) Y. Hamada, T. Sano, M. Fujita, T. Fujii, Y. Nishio and K. Shibata, Jpn. J. Appl. Phys., Part 2, 1993, 32, 514; (c) Y. Shirota, Y. Kuwabara, D. Okuda, R. Okuda, H. Ogawa, H. Inada, T. Wakimoto, H. Nakada, Y. Yonemoto, S. Kawami and K. Imai, J. Lumin., 1997, 72-74, 985.

12 S. A. VanSlyke, C. H. Chen and C. W. Tang, Appl. Phys. Lett., 1996, 69, 2160.

13 K. L. Tong, S. K. So, H. F. Ng, L. M. Leung, M. Y. Yeung and C. F. Lo, Synth. Met., 2004, 147, 199.

14 J. A. Cheng and P. J. Cheng, J. Chem. Crystallogr., 2010, 40, 557.

15 J. D. Luo, Z. L. Xie, J. W. Y. Lam, L. Cheng, H. Y. Chen, C. F. Qiu, H. S. Kwok, X. W. Zhan, Y. Q. Liu, D. B. Zhu and B. Z. Tang, Chem. Commun., 2001, 1740.

16 (a) Y. N. Hong, J. W. Y. Lam and B. Z. Tang, Chem. Commun., 2009, 4332; (b) Y. N. Hong, J. W. Y. Lam and B. Z. Tang, Chem. Soc. Rev., 2011, 40, 5361.

17 Z. J. Zhao, S. M. Chen, J. W. Y. Lam, C. K. W. Jim, C. Y. K. Chan, Z. M. Wang, P. Lu, C. M. Deng, H. S. Kwok, Y. G. Ma and B. Z. Tang, J. Phys. Chem. C, 2010, 114, 7963.

18 Z. J. Zhao, S. M. Chen, C. M. Deng, J. W. Y. Lam, C. Y. K. Chan, P. Lu, Z. M. Wang, B. B. Hu, X. P. Chen, P. Lu, H. S. Kwok, Y. G. Ma, H. Y. Qiu and B. Z. Tang, J. Mater. Chem., 2011, 21, 10949.

19 W. F. Xie, Y. Zhao, C. A. Li and S. Y. Liu, Semicond. Sci. Technol., 2005, 20, 57.

20 A. Saitoh, N. Yamada, M. Yashima, K. Okinaka, A. Senoo, K. Ueno, D. Tanaka and R. Yashiro, SID Int. Symp. Dig. Tech. Pap., 2004, 35, 150. 